\title{
The relative stress on the Achilles tendon during ambulation in an ankle immobiliser: implications for rehabilitation after Achilles tendon repair
}

\author{
K H Akizuki, E J Gartman, B Nisonson, S Ben-Avi, M P McHugh
}

\begin{abstract}
Background-After Achilles tendon repair, immediate weightbearing and immobilisation closer to neutral plantarflexion are thought to limit atrophy and stiffness, but may place deleterious stress on the repair. Objectives-To estimate the relative stress on the Achilles tendon during weightbearing with immobilisation in varying degrees of plantarflexion.

Methods-Electromyographic (EMG) activity from the plantarflexors was recorded during walking in 10 subjects (six men, four women) without ankle pathology. Four walking conditions were examined: (a) normal walking; (b) immobilised (cam-walker) in neutral plantarflexion; (c) immobilised with a 0.5 inch heel lift; (d) immobilised with a 1 inch heel lift. EMG activity relative to plantarflexor torque was determined for each subject during isometric contractions at $\mathbf{2 5 \%}$, $50 \%, 75 \%$, and $100 \%$ of maximal voluntary contraction (MVC). EMG activity during walking was calculated as a percentage of MVC based on the EMG-torque relation during graded isometric contractions.

Results-During normal walking, the plantarflexor torque was estimated to be 30 (12)\% (mean (SD)) of MVC, compared with 21 (15)\% MVC for immobilisation in neutral $(p<0.05), 17$ (15)\% MVC with the addition of a 0.5 inch heel lift $(p<0.01)$, and $12(12) \%$ MVC with the addition of a 1 inch heel lift $(p<0.01)$. The 1 inch heel lift resulted in less than $10^{\circ}$ plantarflexion in all subjects.

Conclusions-When the ankle is immobilised, stress on the Achilles tendon is determined by the degree of plantarflexion and the contractile activity of the plantarflexors. In the immobilised ankle, the addition of a 1 inch heel lift was sufficient to minimise plantarflexor activity during walking.

(BrF Sports Med 2001;35:329-334)
\end{abstract}

Keywords: Achilles tendon; EMG; heel lifts; soleus; gastrocnemius

Although there is still some debate about open versus closed management of Achilles tendon disruption, ${ }^{12}$ most authors agree that surgical repair is the treatment of choice. Until recently, surgical repair followed by cast immobilisation in plantarflexion and non-weightbearing for six weeks was considered the ideal treatment. ${ }^{2}$ However, new controversy has arisen from recent studies that show that early mobilisation seems to enhance the healing of the repair..$^{3-9}$ Many authors are now advocating early range of motion (ROM) based on animal $1^{10-14}$ and hand studies $^{15}$ that show increased healing rates and strength while preventing the side effects of prolonged joint immobilisation, such as joint stiffness, muscular atrophy, cartilage atrophy, deep vein thrombosis, tendocutaneous adhesions, skin necrosis, and disuse osteoporosis. ${ }^{281617}$ If the tendon experiences tension during healing, orientation of collagen fibres and strength of the calf muscles are improved, as are tendon vascularity, ${ }^{11-13} 18$ breaking strength, and number of collagen filaments. ${ }^{19}$ With early ROM and weightbearing protocols, patients are obtaining power and strength that is almost equal to that of the opposite good leg. ${ }^{358}$ By contrast, repair followed by six weeks of cast immobilisation results in appreciable residual weakness. ${ }^{120-22}$ Early ROM and progressive weightbearing appear to result in few failures or complications. ${ }^{53-25}$ However, there is limited clinical research available on which to base the timing and progression of rehabilitation after Achilles tendon repair. For example, cam-walkers are commonly used to provide protected weightbearing by immobilising the ankle in varying degrees of plantarflexion. However, the degree of protection afforded to the Achilles tendon with the use of such immobilisers is not known.

When the ankle is immobilised, stress on the Achilles tendon during gait is determined by the degree of plantarflexion and the contractile activity of the plantarflexors. The degree of plantarflexion can be controlled by using a ROM adjustable cam-walker. Alternatively, cam-walkers with the axis fixed at $90^{\circ}$ can be used, and increased plantarflexion can be achieved by inserting heel lifts into the camwalker. The magnitude of contractile activity during ambulation in a cam-walker can be quantified with surface electromyography (sEMG). Therefore, the purpose of this study was to use sEMG to estimate the relative stress on the Achilles tendon during weightbearing with immobilisation in varying degrees of plantarflexion using heel lifts inserted into a cam-walker.

\section{Methods}

EXPERIMENTAL PROTOCOL

Ten subjects (six men, four women; age 28 (6) years; height $173(10) \mathrm{cm}$; mass $77.4(16.6) \mathrm{kg}$; mean (SD)) without known ankle pathology volunteered to be examined in four walking conditions: (a) normal walking; (b) immobilised (cam-walker) in neutral plantarflexion; 
A

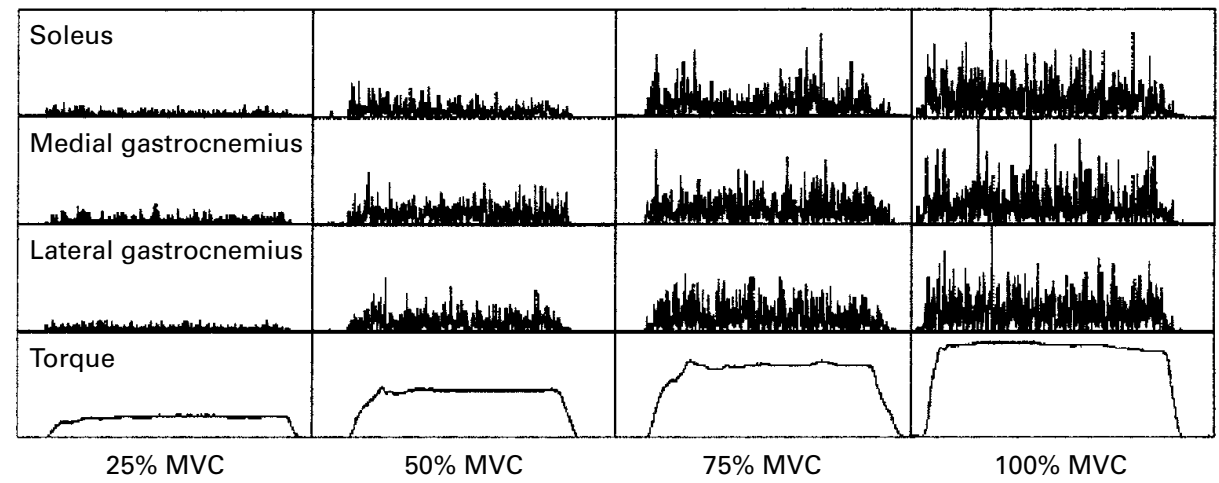

B Torque $=0.15 \mathrm{RMS}+7.9$

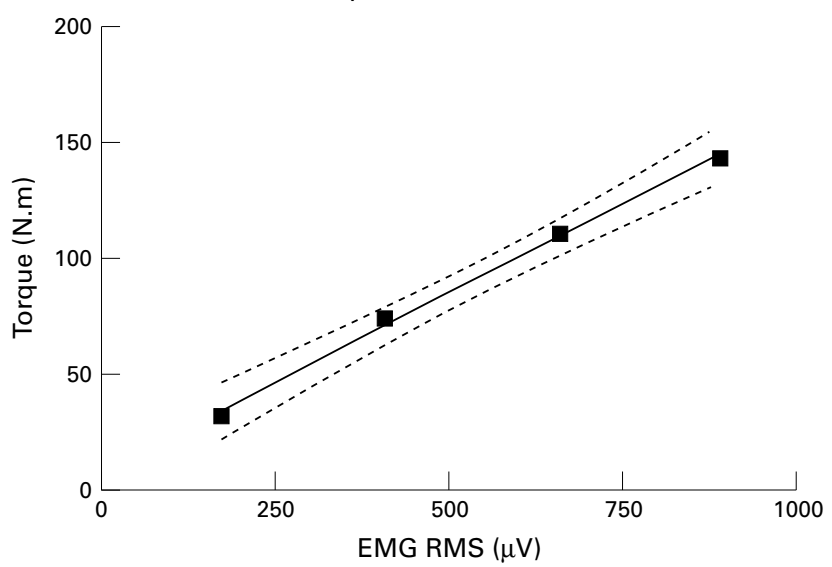

Figure 1 (A) Rectified electromyographic (EMG) signals and torque from selected isometric plantarflexion contractions at each intensity in one subject (a total of three contractions were performed at each intensity). The peak torque and the peak value from the root mean square (RMS) of the EMG signal were recorded. (B) Linear regression (95\% confidence intervals shown by dashed line) of plantarflexor EMG activity ( $x$ axis) versus plantarflexion torque (y axis) for the subject whose raw data is shown in $(A)$.

(c) immobilised with a 0.5 inch heel lift; $(d)$ immobilised with a 1 inch heel lift. The actual degree of plantarflexion was calculated on the basis of the height of the heel lift and the distance from the metatarsal heads to the calcaneus. For example, inserting a 1 inch heel lift under the calcaneus of a foot with a distance of 6 inches from the third metatarsal head to the centre of the calcaneus would create $9.6^{\circ}$ of plantarflexion $\left(\operatorname{sine}^{-1}(1 / 6)\right)$. A force-sensitive resistor (foot switch) was placed on the sole of the cam-walker to indicate heel strike and toe off. Each walking condition was tested twice for a total of eight trials. The test sequence for the eight trials was as follows: trial 1, normal walking; trial 2, immobilised in neutral plantarflexion; trial 3, immobilised with a 0.5 inch heel lift; trial 4, immobilised with a 1 inch heel lift; trial 5, immobilised with a 1 inch heel lift; trial 6, immobilised with a 0.5 inch heel lift; trial 7 , immobilised in neutral plantarflexion; trial 8, normal walking. The test sequence was reversed for the second set of trials to control for any adaptation to the previous trials. In each condition, the subject walked for 10 steps (five steps on the instrumented leg).
EMG TECHNIQUES

During the walking conditions, EMG activity was recorded from surface electrodes (Ag/ $\mathrm{AgCl})$ placed over the medial gastrocnemius, lateral gastrocnemius, and soleus muscles. The skin was shaved, cleaned, and abraded before application of $10 \mathrm{~mm}$ diameter $\mathrm{Ag} / \mathrm{AgCl}$ electrodes on a $34 \mathrm{~mm}$ by $22 \mathrm{~mm}$ adhesive gel surface. For the medial gastrocnemius and lateral gastrocnemius, pairs of electrodes were placed longitudinally $3 \mathrm{~cm}$ apart, one handbreadth below the popliteal crease on the medial and lateral masses of the respective muscles. For the soleus, a pair of electrodes was placed longitudinally $3 \mathrm{~cm}$ apart immediately distal to the gastrocnemius bellies and medial to the Achilles tendon.

The EMG signal was recorded by telemetry, bandpass filtered from 12 to $500 \mathrm{~Hz}$, and sampled at a rate of $1000 \mathrm{~Hz}$ with a common mode rejection ratio of $135 \mathrm{~dB}$ (Noraxon, Scottsdale, Arizona, USA). The amplitude of the EMG signal was quantified by computing the root mean square (RMS) of the raw signal with sliding 50 millisecond windows. This provided a smoothed curve from which a peak value could be identified. The peak value during 


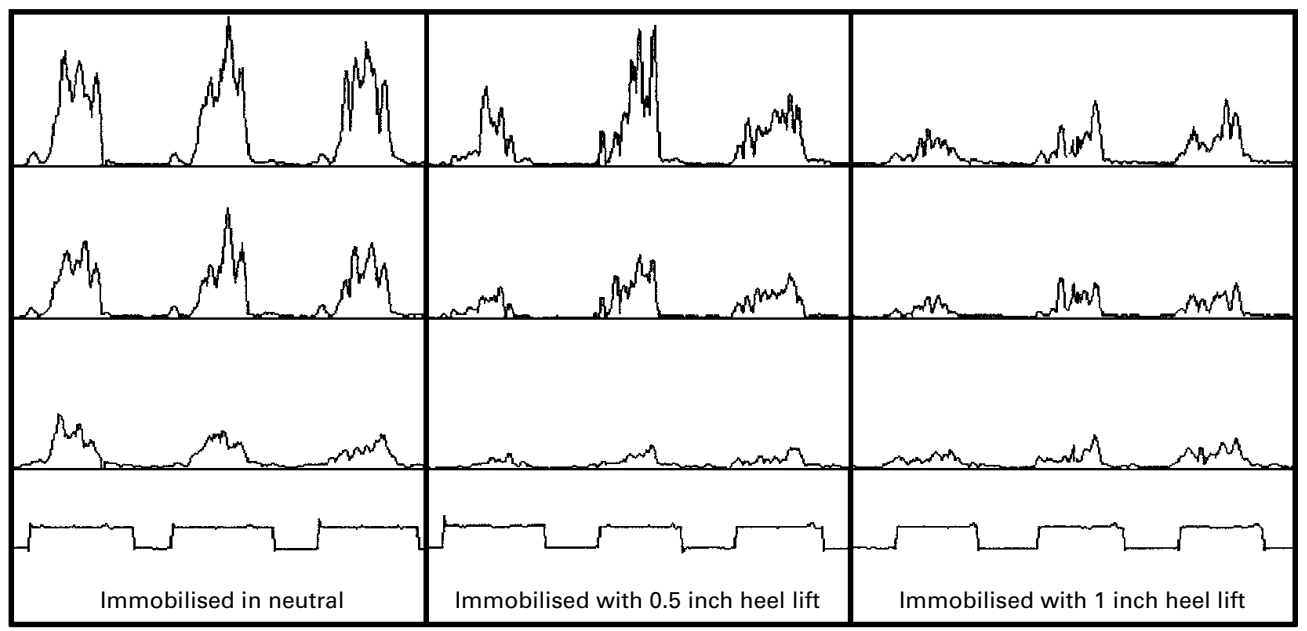

Figure 2 Root mean square of the plantarflexor electromyographic (EMG) signals for the three immobilised conditions in a selected subject. Three steps are shown for each condition. The top tracing is the soleus, the middle tracing is the medial gastrocnemius, the third tracing is the lateral gastrocnemius, and the bottom tracing is the foot switch indicating ground contact. The scale on the EMG channels is $250 \mu \mathrm{V}$. A progressive decrease in EMG activity with addition of heel lifts is evident.

ground contact was recorded for each step, summed for each muscle, and averaged for five steps in each condition.

\section{TECHNIQUE FOR ESTIMATING PLANTARFLEXION} TORQUE IN CAM-WALKER

After the walking trials, subjects performed isometric plantarflexion contractions on a dynamometer during which EMG activity was recorded. Subjects were placed in a semireclined position, with the hip flexed to $20^{\circ}$, the knee flexed to $30^{\circ}$, and the ankle at $90^{\circ}$. Three maximum isometric contractions (MVC) were performed. The subjects then performed three contractions at $25 \%, 50 \%$, and $75 \%$ of MVC. Each contraction lasted three seconds with 10 seconds between contractions and two minutes between sets of three. The RMS of the raw EMG signal was computed for each contraction and averaged for each intensity (fig 1A). Linear regression equations of RMS versus plantarflexion torque, at each intensity, were computed for each subject (fig 1B). Using the regression equations, the EMG activity recorded during the four walking conditions was converted into torque values. These estimated torque values were then expressed as a percentage of MVC torque.

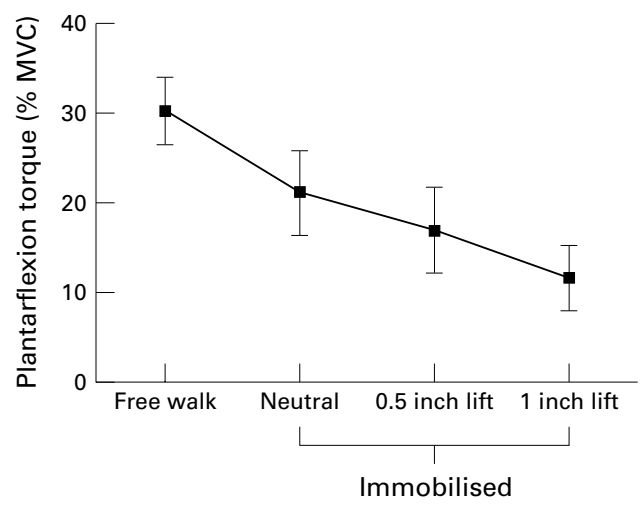

Figure 3 Estimated plantarflexion torque for each of the four walking conditions. There was a progressive decrease in plantarflexion torque with immobilisation and subsequent addition of heel lifts $(p<0.001)$.
STATISTICAL ANALYSIS

The summed EMG values were averaged for the two trials in each walking condition, and repeated measures analysis of variance was used to examine the effect of walking condition on EMG amplitude (RMS). The effects of the different walking conditions on the estimated plantarflexion torque (calculated from the EMG-torque regression equations) and estimated percentage of maximum plantarflexion torque (\% MVC torque) were also tested using repeated measures analysis of variance. Bonferroni corrections were applied to all pairwise comparisons between the four walking conditions.

\section{Results}

There was a progressive decrease in plantarflexor EMG activity with each walking condition $(p<0.001)$. Walking with the ankle immobilised in neutral plantarflexion reduced plantarflexor EMG activity by $21 \%$ compared with normal walking $(\mathrm{p}<0.05)$. Adding a 0.5 inch heel lift further reduced EMG activity by $31 \%$ relative to normal walking $(\mathrm{p}<0.01)$; this was $13 \%$ lower than activity when immobilised in neutral $(p=0.41)$. The 1 inch heel lift reduced plantarflexor activity by $43 \%$ relative to normal walking $(p<0.01)$; this was $22 \%$ lower than activity when immobilised in neutral $(p<0.05)$ and $12 \%$ lower than activity when immobilised with a 0.5 inch heel lift $(p=0.5)$. The 1 inch heel lift resulted in less than $10^{\circ}$ plantarflexion in all subjects. Figure 2 shows a typical sEMG recording of the three immobilised conditions. Of note is that the stance phase was about $60 \%$ of the gait cycle in each of the three immobilised conditions. Therefore normal timing of gait was not changed.

During normal walking, the plantarflexor torque was estimated to be $30(12) \%$ (mean (SD)) of MVC, compared with 21 (15)\% of MVC for immobilisation in neutral $(\mathrm{p}<0.05 v$ normal walking), 17 (15)\% of MVC with the addition of a 0.5 inch heel lift $(p<0.01 v$ normal walking; $\mathrm{p}=0.56 \mathrm{v}$ neutral), and 12 (12)\% of MVC with the addition of a 1 inch 
heel lift $(\mathrm{p}<0.001 v$ normal walking; $\mathrm{p}<0.05 v$ neutral; $\mathrm{p}=0.36 v 0.5$ inch; fig 3). Estimated plantarflexion torques were 36 (12) N.m, 24 (14) N.m, 18 (16) N.m, and 12 (12) N.m respectively in each of the four walking conditions.

\section{Discussion}

Stress on the repaired Achilles tendon can be avoided by immobilising the ankle in a position in which the tendon is on slack. The actual angle at which this occurs depends on how well the ends were approximated at the time of surgery. However, the greater the degree of plantarflexion in which the ankle is immobilised the greater will be the subsequent atrophy. ${ }^{16}{ }^{17}$ Furthermore, non-weightbearing and prolonged disuse facilitate atrophy and stiffness. ${ }^{16}{ }^{17}$ On the other hand, contractile activity during weightbearing on the immobilised ankle may result in undesirable stresses on the Achilles. The present data clearly show that, in normal ankles, there is still significant contractile activity in the plantarflexors with immobilisation in neutral plantarflexion $(79 \%$ of normal walking). The simple addition of a 1 inch heel lift was sufficient to reduce this contractile activity to $57 \%$ of normal walking while producing less than $10^{\circ}$ of plantarflexion.

Although immobilisation in minimal plantarflexion may successfully reduce plantarflexor activity, it is not known how much stress this reduced activity places on the Achilles tendon, and, more importantly, what magnitude of activity represents a safe amount of stress on a repair. Benum et $a l^{26}$ estimated Achilles forces during walking with the ankle casted at $20^{\circ}$ of plantarflexion in five patients two weeks after Achilles tendon repair. The relation of EMG activity to torque during dynamometric testing of the non-involved plantarflexors was used to estimate torques in the immobilised ankle during walking. Achilles forces were then estimated on the basis of plantarflexion torques and the distance from the centre of rotation of the ankle and the line of pull of the Achillesthat is, the lever arm. Achilles forces were estimated to be $104 \mathrm{~N}$ during walking two weeks after surgery. The patients had previously used crutches for partial weightbearing and were subsequently allowed to fully weightbear. However, given the degree of plantarflexion, patients had to walk with the leg in external rotation (only two patients could walk normally). Using the same method of calculation, Achilles forces during the four walking conditions in this study were estimated to be 553 (182) N during normal walking, 369 (252) N immobilised in neutral, 282 (212) $\mathrm{N}$ immobilised with a 0.5 inch heel lift, and 191 (188) N immobilised with a 1 inch heel lift. The higher estimates of Achilles forces compared with those of Benum et $a l^{26}$ can be attributed to testing in less plantarflexion and using normal subjects without atrophy.

The question of whether about $200 \mathrm{~N}$ of force is safe depends on factors such as the strength of the repair, the effect of cyclic loading, the timing of the weightbearing (how soon after surgery), and the rate of tendon healing.
Previous reports indicate that the tensile strength of cadaveric Achilles repairs is significantly less than $200 \mathrm{~N}(45-175 \quad \mathrm{~N}) .^{27} 28$ However, the strength of the healing tendon would need to be more than twice the load imposed during gait to avoid the detrimental effects of cyclic loading. Therefore, from this approximation of Achilles forces, stronger surgical repairs may be needed or significant tendon healing may be required before progressing to full weightbearing. The clinical dilemma is that no stress accelerates atrophy whereas too much stress may jeopardise the repair. However, these data do not provide an insight into what conditions provide the optimal stress in the period immediately after surgery.

Aoki et $a l^{23}$ had patients full weightbearing in neutral two to three weeks after surgery. Two of 22 patients had partial ruptures within eight weeks of surgery. Speck et $a l^{4}$ allowed "early full weightbearing" at one day after surgery, with the ankle immobilised in neutral. However, it was not clear whether the patients were full weightbearing all of the time or just for selected rehabilitation exercises. None of the 20 patients experienced reruptures and had good functional results. Considering the estimations of repair strengths and Achilles forces during gait, these results are surprising. It is possible that neural inhibition of the plantarflexors secondary to pain and swelling results in lower Achilles forces than estimated here. However, it is also important to note that previous studies ${ }^{29} 3031$ using force transducers implanted into the Achilles tendon reported forces well in excess of the forces estimated in the present study. A recent study using an optic fibre technique recorded peak Achilles forces during walking of about $1300 \mathrm{~N} .^{31}$ The experimental approach used here was to relate EMG amplitudes during ambulation to joint torques based on isometric testing. The accuracy of this approach comes from the fact that the plantarflexors are primarily working isometrically when the ankle is immobilised. The prediction of ankle torques and Achilles forces during normal walking (without immobilisation) is inherently less accurate using this approach because the contractions are no longer isometric.

Some cam-walkers can be adjusted to manipulate ankle angle thereby affecting the position of immobilisation. This is useful for treating Achilles tendon repairs because immobilisation in plantarflexion will decrease the mechanical stress on the repair. However, after preliminary experimentation, we chose to use heel lifts to provide increased plantarflexion rather than manipulate the cam-walker. This was done for three reasons: first, rotating the cam-walker into plantarflexion forced the patient into a toe touch gait resulting in a more awkward gait necessitating some rotation at the hip; second, the goal was to reduce contractile activity without significantly shortening the muscle length; third, if the heel lifts were shown to be effective, this would negate the need to purchase the more expensive adjustable immobiliser. The results support the use of heel lifts to reduce plantarflexor activity. 
In summary, immobilising the ankle in a cam-walker and inserting a 1 inch heel lift was sufficient to reduce plantarflexor sEMG activity to $57 \%$ of the activity during normal walking. It was estimated that this represented a plantarflexion torque of $12 \%$ of MVC. Although the 1 inch heel lift resulted in less than $10^{\circ}$ of plantarflexion, it reduced plantarflexor sEMG activity by $22 \%$ relative to immobilisation in neutral. After Achilles tendon repair, heel lifts can be used as an inexpensive means of reducing stress on the repair while progressing a patient's weightbearing status.

1 Inglis AE, Scott WN, Sculco TP, et al. Rupture of the tendoachilles: an objective assessment of surgical and nonsurgical treatment. F Bone foint Surg [Am] 1976;58:990-3.

2 Wills CA, Washburn S, Caiozzo V, et al. Achilles tendon rupture. A review of the literature comparing surgical and non-surgical treatment. Clin Orthop 1986;207:156-63.

3 Solveborn SA, Moberg A. Immediate free ankle motion after surgical repair of acute Achilles tendon ruptures. $A m$ f Sports Med 1994;22:607-10.

4 Cetti R. Ruptured Achilles tendon: preliminary results of a new treatment. Br $\mathcal{F}$ Sports Med 1988;22:6-8.

5 Mandelbaum BR, Myerson MS, Forster R. Achilles tendon ruptures: a new method of repair, early range of motion, ruptures: a new method of repair, early range of motion, 392-5.

6 Carter TR, Fowler PJ, Blokker C. Functional postoperative treatment of Achilles tendon repair. Am F Sports Med 1992 20:459-62

7 Troop RL, Losse GM, Lane JG, et al. Early motion after Achilles tendon ruptures. Foot Ankle Int 1995;16:705-8.

8 Saw Y, Baltzopoulos V, Lim A, et al. Early mobilization after operative repair of ruptured Achillles tendon. Injury 1993 24:479-84.

9 Mortensen NHM, Skov O, Jensen PE. Early motion of the ankle after operative treatment of a rupture of the Achilles tendon. F Bone foint Surg [Am] 1999;81:983-90.

10 Peacock, E. Biological principles in the healing of long tendons. Surg Clin North Am 1965;45:461.

11 Gelberman, RH, Menon, J, Gonsalves M, et al. The effects of mobilization on the vascularization of healing flexor tendons in dogs. Clin Orthop 1980;153:283-9.

12 Gelberman RH, Manske PR, Vande Berg JS, et al. Flexor tendon repair in-vitro: a comparative histologic study of the rabbit, chicken, dog and monkey. F Orthop Res 1984;2:3948 .
13 Gelberman RH, Woo SL-Y, Lothringer K, et al. Effects of early intermittent passive mobilization on healing canine flexor tendons. F Hand Surg 1982;7:170-5.

14 Gelberman RH, Vande Berg JS, Lunborg GN, et al. Flexor tendon healing and restoration of the gliding surface. $f$ Bone foint Surg [Am] 1983;65:70-80.

15 Crosby CA, Wehbe MA. Early motion protocols in hand and wrist rehabilitation. Hand Clin 1996;12:31-41.

16 Booth FW. Physiologic and biochemical effects of immobilization on muscle. Clin Orthop 1987;219:15-20.

17 Booth FW. Effect of limb immobilization on skeletal muscle. f Appl Physiol. 1982;52:1113-18.

18 Rothman RH, Slogoff S. The effect of immobilization on the vascular bed of tendon. Surg Gynecol Obstet 1967;124: 1064-6.

19 Enwemeka CS, Spielholz NI, Nelson AJ. The effect of early functional activities on experimentally tenotomized Achilles tendons in rats. Am 7 Phys Med Rehabil 1988;67:264-9.

20 Nistor L. Surgical and non-surgical treatment of achilles tendon rupture. F Bone foint Surg [Am] 1981;63:394-9.

21 Bradley JP, Tibone JE. Percutaneous and open surgical repairs of achilles tendon ruptures: a comparative study. Am f Sports Med 1990;18:188-95.

22 Shields CL, Kerlan RK, Jobe FW, et al. the cybex II evaluation of surgically repaired achilles tendon ruptures. $A m 7$ Sports Med 1978;6:369-72.

23 Aoki M, Ogiwara N, Ohta T, et al. Early active motion and weightbearing after cross-stitch Achilles tendon repair. Am 7 Sports Med 1998;26:794-800.

24 Speck M, Klaue K. Early full weightbearing and functional treatment after surgical repair of acute Achilles tendon rupture. Am f Sports Med 1998;26:789-93.

25 Soma C, Mandelbaum BR. Repair of acute Achilles tendon ruptures. Orthop Clin North Am 1995;26:239-47.

26 Benum P, Berg V, Fretheim OJ. The strain on sutured Achilles tendons in walking cast: An EMG analysis. Eur Surg Res 1984;16(suppl):14-21

27 Mortensen NHM, Saether J. Achilles tendon repair: a new method of Achilles tendon repair tested on cadaverous materials. F Trauma 1991;31:381-4.

28 Watson TW, Jurist KA, Yang KH, et al. The strength of Achilles tendon repair: an in vitro study of the biomechaniAchilles tendon repair: an in vitro study of the biomechani1995; 16:191-5.

29 Komi PV, Solonen M, Järvinen M, et al. In vivo registration of Achilles tendon forces in man. I. Methodological development. Int $\mathcal{F}$ Sports Med 1987;8:3-8.

30 Komi PV, Fukashiro S, Järvinen M. Biomechanical loading of Achilles tendon during normal locomotion. Clin Sports Med 1992;11:521-30.

31 Finni T, Komi PV, Lukkariniemi J. Achilles tendon loading during walking: application of a novel optic fiber technique. Eur F Appl Physiol 1998;77:289-91.

\section{Take home message}

When the ankle is immobilised in the neutral position, there is still significant contractile activity in the plantarflexors during ambulation. However, the simple addition of a 1 inch heel lift can be used to reduce this contractile activity considerably.

\section{Commentary}

Increasing plantarflexion of the ankle decreases the stress on the Achilles tendon during ambulation in an ankle immobiliser, but how much stress is optimal in the various phases after an Achilles injury?

The atrophying effects of immobilisation and the strengthening effects of training and exercise on all musculoskeletal tissues (bone, cartilage, ligament, muscle, and tendon) are well known, and therefore much effort has been expended during recent decades on studies to clarify whether the old concept of post-traumatic immobilisation could be safely replaced by rehabilitation programmes using early controlled mobilisation. The controlled experimental and clinical trials have yielded convincing evidence that early controlled mobilisation is superior to immobilisation for musculoskeletal soft tissue injuries. ${ }^{12}$ This holds true not only in primary treatment of acute injuries, but also in their postoperative management. The superiority of early controlled mobilisation is especially apparent in producing quicker recovery and return to full activity, without jeopardising the long term rehabilitation.

Although the above general message is clear, many interesting problems with respect to the content and timing of controlled rehabilitation need further clarification. For example, clear answers are needed on the effects of various joint positions on the mechanical stress of injured and repaired tendons and ligaments. This study addresses one of these problems: using surface electromyography and isometric torque measurements, the authors determined the relative stresses 
on the Achilles tendon during weightbearing with immobilisation in varying degrees of ankle plantarflexion. As the result of carefully conducted measurements with 10 adult subjects, they calculated that the Achilles forces averaged about $550 \mathrm{~N}$ during normal walking, $370 \mathrm{~N}$ during walking with the ankle immobilised in neutral position, $280 \mathrm{~N}$ during walking with the ankle immobilised with a 0.5 inch heel lift, and $190 \mathrm{~N}$ when walking with the ankle immobilised with a 1 inch heel lift. This result - that is, immobilisation with increasing plantarflexion of the ankle decreases the EMG activity and force of the calf muscles and thus the resultant Achilles tendon strain during walking - is not surprising as it follows common sense and the basic rules of musculoskeletal biomechanics. It should be of use if, after an Achilles tendon injury, reduction of stress is desired while progressing with the patient's weightbearing.

What remains a challenge is the question about the limits of desirable and undesirable stresses on the Achilles tendon during various phases of healing. As complete immobility with zero stress leads to tissue atrophy and adhesions whereas too much stress too early is likely to lengthen the healing tissue and jeopardise the repair, we need to know much more about the optimal tendon strains and rehabilitation after an Achilles tendon injury.

Professor of Injury Prevention, Department of Surgery,

P KANNUS

Tampere University Medical School and University Hospital, Tampere, Finland

1 Jozsa L, Kannus P. Human tendons: anatomy, physiology, and pathology. Champaign IL: Human Kinetics, 1997:1-574.

2 Kannus P. Immobilisation or early mobilisation after an acute soft-tissue injury? Physician and Sports Medicine 2000;29:55-63.

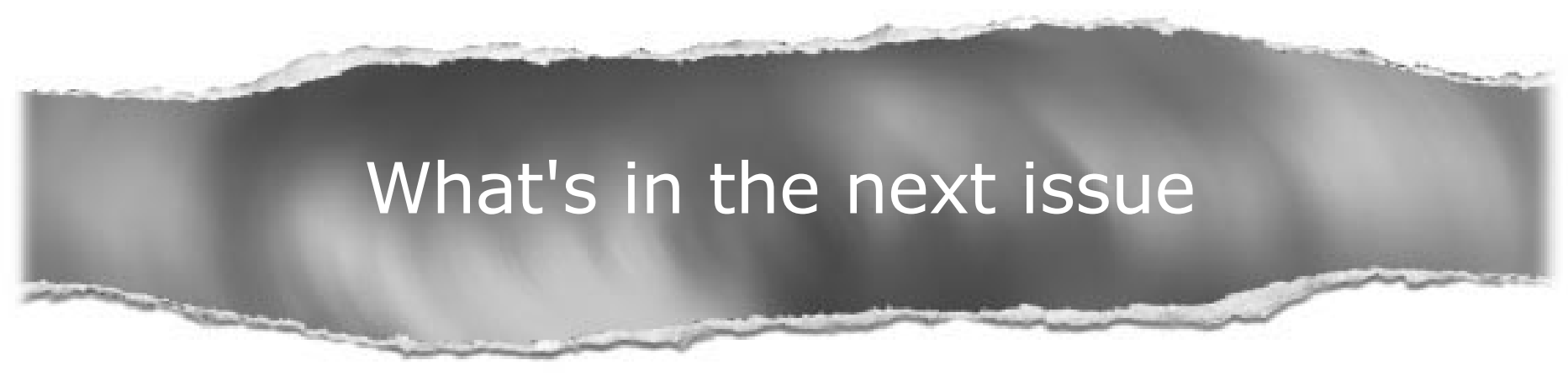

Future content

See which articles have just been accepted for publication and preview the table of contents for the next issue a month before it is published

www.bjsportmed.com 